\title{
Surgically resected skull base meningiomas demonstrate a divergent postoperative recurrence pattern compared with non-skull base meningiomas
}

\author{
Alireza Mansouri, MD, ${ }^{1-3}$ George Klironomos, MD, PhD, ${ }^{1,2}$ Shervin Taslimi, MD, MPH, ${ }^{1,2}$ \\ Alex Kilian, ${ }^{2}$ Fred Gentili, MD, MSc, ${ }^{1,2}$ Osaama H. Khan, MD, ${ }^{1,2}$ Kenneth Aldape, MD, ${ }^{4}$ and \\ Gelareh Zadeh, MD, PhD',2 \\ ${ }^{1}$ Division of Neurosurgery, University of Toronto; ' ${ }^{2}$ epartment of Neurosurgery, Toronto Western Hospital, University Health \\ Network, Toronto; ${ }^{3}$ Department of Clinical Epidemiology and Biostatistics, McMaster University, Hamilton; and ${ }^{4}$ Department of \\ Pathology, University of Toronto, Ontario, Canada
}

\begin{abstract}
OBJECTIVE The objective of this study was to identify the natural history and clinical predictors of postoperative recurrence of skull base and non-skull base meningiomas.

METHODS The authors performed a retrospective hospital-based study of all patients with meningioma referred to their institution from September 1993 to January 2014. The cohort constituted both patients with a first-time presentation and those with evidence of recurrence. Kaplan-Meier curves were constructed for analysis of recurrence and differences were assessed using the log-rank test. Cox proportional hazard regression was used to identify potential predictors of recurrence.
\end{abstract}

RESULTS Overall, 398 intracranial meningiomas were reviewed, including 269 (68\%) non-skull base and $129(32 \%)$ skull base meningiomas (median follow-up 30.2 months, interquartile range [IQR] 8.5-76 months). The 10-year recurrence-free survival rates for patients with gross-total resection (GTR) and subtotal resection (STR) were $90 \%$ and $43 \%$, respectively. Skull base tumors were associated with a lower proliferation index ( 0.041 vs $0.062, p=0.001$ ), higher likelihood of WHO Grade I ( $85.3 \%$ vs $69.1 \%, p=0.003)$, and younger patient age ( 55.2 vs 58.3 years, $p=0.01)$. Meningiomas in all locations demonstrated an average recurrence rate of $30 \%$ at 100 months of follow-up. Subsequently, the recurrence of skull base meningiomas plateaued whereas non-skull base lesions had an $80 \%$ recurrence rate at 230 months follow-up $(p=0.02)$. On univariate analysis, a prior history of recurrence $(p<0.001)$, initial WHO grade following resection $(p<0.001)$, and the inability to obtain GTR $(p<0.001)$ were predictors of future recurrence. On multivariate analysis a prior history of recurrence $(p=0.02)$ and an STR $(p<0.01)$ were independent predictors of a recurrence. Assessing only patients with primary presentations, STR and WHO Grades II and III were independent predictors of recurrence $(p<$ 0.001 for both).

CONCLUSIONS Patients with skull base meningiomas present at a younger age and have less aggressive lesions overall. Extent of resection is a key predictor of recurrence and long-term follow-up of meningiomas is necessary, especially for non-skull base tumors. In skull base meningiomas, recurrence risk plateaus approximately 100 months after surgery, suggesting that for this specific cohort, follow-up after 100 months can be less frequent.

http://thejns.org/doi/abs/10.3171/2015.7.JNS15546

KEY WORDS meningioma; postoperative period; recurrence; skull base

$\mathrm{M}$ ENINGIOMAS are the second most common primary intracranial tumors in adults. ${ }^{32}$ These tumors tend to exhibit a slow growth rate and minimal invasion of the brain parenchyma; therefore, they are typically considered benign. The optimal management of meningiomas is complete resection of the tumor along with the associated dura and bone, traditionally recognized as a Simpson Grade I resection. ${ }^{30}$ However, this goal cannot always be achieved safely. This is particularly true for meningiomas in regions that are associated with critical neurovascular structures, typically in the skull base. Also, it has been suggested that even upon achieving an apparent

ABBREVIATIONS $\mathrm{Cl}$ = confidence interval; EOR = extent of resection; $\mathrm{GTR}$ = gross-total resection; IQR = interquartile range; NF2 = neurofibromatosis Type 2; OR = odds ratio; $P R=$ progesterone receptor; STR = subtotal resection.

SUBMITTED March 8, 2015. ACCEPTED July 15, 2015.

INCLUDE WHEN CITING Published online January 1, 2016; DOI: 10.3171/2015.7.JNS15546. 
gross-total resection (GTR), microscopic remnants may remain adherent to neurovascular structures, resulting in recurrence. ${ }^{2,6,21}$ Studies assessing the long-term outcomes of surgically resected meningiomas suggest that the recurrence rate is a clinically relevant concern, despite the benign nature of the majority of meningiomas, particularly for subtotally resected lesions, and follow-up beyond 10-20 years is required. ${ }^{19,30}$

The majority of surgical series assessing the long-term recurrence patterns of meningiomas have focused on the extent of resection (EOR). However, recent studies have suggested that in addition to the EOR, the pattern of meningioma recurrence may be dependent on the tumor biology as well. While the proliferation index (MIB-1 index) has been recognized as a relevant histological marker, the correlation of the mitotic index based on phosphohistone H3 staining and recurrence-free survival has also been noted..$^{9,20,26}$ A growing body of literature, with expanding population cohorts followed over longer periods of time, suggests that skull base meningiomas may, in fact, be genetically different from their non-skull base counterparts.,20,29 While many studies suggest that skull base meningiomas have lower proliferative indices, the evidence correlating this information with recurrence rates has been inconsistent. ${ }^{9,14,20}$

In the current study, we report on a consecutive series of patients with surgically managed meningiomas to determine whether skull base meningiomas demonstrate a natural history that is different from non-skull base meningiomas. In addition, we have sought to explore the interplay of EOR with additional factors that could be predictors of postoperative tumor recurrence as well. Awareness of the recurrence patterns of meningiomas over the long term, as determined by patient and tumor characteristics, would help determine the optimal frequency and duration of follow-up and guide the choice of postoperative intervention for residuals and recurrences.

\section{Methods}

\section{Patients and Setting}

Following institutional ethics board approval, a retrospective analysis of the database on surgically treated meningiomas was conducted. All consecutive patients referred to our hospital for surgical management of meningiomas from September 1993 to January 2014 were analyzed, including patients in whom a prior surgical intervention had been undertaken elsewhere. Only patients with a minimum of 6 months of follow-up with available clinical, imaging, and pathological data were included. Baseline patient demographic data were collected. Patients with a diagnosis of neurofibromatosis Type 2 (NF2) were also excluded.

\section{Definition of Tumor Location}

Tumors located in the anterior/middle cranial fossa (including the olfactory groove, tuberculum sella, planum sphenoidale, anterior clinoid, and sphenoid ridge), cavernous sinus, petroclival/clival area, basal foramina (e.g., jugular foramen), and the petrous bone were classified as skull base. Meningiomas located on the sphenoid ridge were further subclassified into medial and lateral, depending on the epicenter of the tumor in relation to the middle of the sphenoid wing. Tumors located in the supratentorial/infratentorial convexity, tentorial region, and parasagittal regions were considered non-skull base. Patients with intraventricular and spinal meningiomas were excluded.

\section{Clinical Patient Information}

Any history of hormone replacement therapy/oral contraceptive medication use, the presence of pre- or postoperative active seizures (not attributable to alternative pathology)/dependence on antiepileptic medications, perioperative antiepileptic therapy, postoperative radiation therapy, remote and/or prior history of radiation, and number of total resections was documented. Time to recurrence was also documented. Any tumor growth resulting in symptomatic changes was considered a recurrence of significance. In asymptomatic cases, tumor recurrence was defined as $\geq 10 \%$ growth in either of greatest diameter or volume, taking whichever value was highest. This threshold was selected to account for possible inaccuracies in measurements. While patient survival was documented, it was not studied as an outcome for analysis given the lack of clarity with regard to the cause of death and the invalidity of assumptions with regard to patients lost to follow-up.

Our institutional practice with regard to postoperative intervention and/or follow-up is as follows: WHO Grade I meningiomas with an uncomplicated resection are followed-up every 6 months for the first year, and annually thereafter with MRI and clinical assessment; imaging follow-up in earlier series of patients was based on CT imaging. WHO Grade II meningiomas with a subtotal resection (STR) are considered for postoperative radiationbased therapy, in consultation with radiation oncology colleagues as part of our institutional tumor board meetings. Considerations toward this decision include the size of the residual tumor, patient age, expected survival, along with additional case-by-case factors. Those with a GTR are followed-up every 6 months for the first 2 years, and annually thereafter. WHO Grade III meningiomas with any residual undergo postoperative radiation therapy, with a decision for stereotactic radiosurgery versus fractionated radiation made based on review at a multidisciplinary tumor board conference. Following radiation treatment, patients are followed every 3-6 months.

\section{Imaging (CT or MRI, Where Available)}

Tumor size (both tumor volume and greatest diameter in a major cardinal plane, or only the latter when volume assessment was not feasible), location, evidence of peritumoral edema and its volume, and evidence of pre- or postoperative hemorrhage (based on the presence of hyperdensity within the tumor resection cavity on postoperative CT) were documented. Volumetric tumor analysis was conducted for cases in which appropriate DICOM images were available on our hospital radiology files. Measurements for both the lesion and the surrounding edema were made using T1-weighted MRI with Gd enhancement and T2-weighted 1.5-T/3-T MRI using ITK-SNAP 2.2 software. The validity of the ITK-SNAP software has been confirmed in the measurement of several anatomi- 
cal structures..$^{10,33}$ Assessment of growth was made at each successive follow-up evaluation compared with the immediate postoperative images.

\section{Tumor Pathology}

Tumor WHO grade (along with grade of recurrent lesion, if relevant), MIB-1 index of first tumor resection at our hospital, and progesterone receptor (PR) status were documented. All of the meningiomas resected prior to 2000 underwent WHO reclassification to provide an updated WHO grade. PR status was recorded based on the classification used by Hsu et al.:11 in that study, patients with no PR staining were found to have the shortest disease-free interval. Patients in our study were dichotomized as either having no PR staining (a score of 0 ) or having scores of 1 to 4 .

\section{Extent of Resection}

The EOR was determined based on operative reports in addition to confirmation based on the first postoperative CT scan and/or MRI. In our analysis, Simpson resection grades were dichotomized: Grade I/II resection was considered a GTR, while higher Simpson grades were considered an STR. ${ }^{19,20,31}$

\section{Statistical Analysis}

Independent t-tests and Mann-Whitney tests were used to compare continuous variables. The chi-square test was used to compare categorical variables. Kaplan-Meier curves were generated to analyze the relationship between tumor recurrence and different variables, including tumor location. The log-rank test was used to assess for significant differences among these curves. Cox proportional hazard regression was used for evaluating different factors that might influence recurrence while controlling for confounders.

Univariate and multivariate analysis was used to identify potential predictors of recurrence. Variables with a $p$ value $<0.2$ on univariate analysis were carried forward to the multivariate analysis. All statistical analyses were 2 -sided and conducted using SPSS (version 22.0, IBM). For the multivariate analysis and all other statistical analyses, a $\mathrm{p}$ value $<0.05$ was considered significant; the Bonferroni correction was applied to account for multiple comparisons, when applicable.

\section{Results}

\section{Baseline Patient Information}

Between September 1993 and January 2014, a total of 465 patients who had complete long-term follow-up data, including clinical and imaging data with a diagnosis of meningioma, were included in this study. A total of 67 patients were excluded from the study: 10 of these patients lacked clinical follow-up data, 8 had intraventricular meningiomas, 39 had spinal meningiomas, and 10 were diagnosed with NF2. Therefore, for this study we had a total of 398 intracranial meningiomas available for review (Table 1).

The median follow-up was 30.2 months (interquartile range [IQR] 8.5-76 months). Among these patients, the majority were female (65.8\%), had tumors in a non-
TABLE 1. Baseline patient- and tumor-related characteristics across all patients analyzed in the database

\begin{tabular}{lc}
\hline \multicolumn{1}{c}{ Characteristic } & Frequency (\%) \\
\hline Non-skull base & $269(67.6)$ \\
\hline Skull base & $129(32.4)$ \\
\hline Males & $136(34.2)$ \\
\hline Median follow-up in mos (IQR) & $30.2(8.5-76)$ \\
\hline Initial WHO grade & \\
I & $296(74.4)$ \\
II & $78(19.6)$ \\
III & $23(5.8)$ \\
Unknown & $1(<0.1)$ \\
\hline Radiation history & $29(7.3)$ \\
\hline Primary presentation at initial op & $359(90.2)$ \\
\hline Preop intratumoral hemorrhage & $8(2)$ \\
\hline Postop tumor bed hemorrhage & $119(29.9)$ \\
\hline Preop seizure & $95(23.9)$ \\
\hline Postop seizure & $70(17.6)$ \\
\hline Periop AEDs & $14(3.5)$ \\
\hline PR status positive (Classes 1-4) & $115(28.9)$ \\
\hline STR & $125(31.4)$ \\
\hline
\end{tabular}

AEDs $=$ antiepileptic drugs.

skull base location (67.6\%), and were found to have a WHO Grade I tumor (74.4\%). One hundred twenty-nine patients (32.4\%) had skull base meningiomas (Table 2). A potential diagnosis of radiation-induced meningioma, based on a history of remote radiation, was made in 29 patients $(7.3 \%)$. Postoperative hemorrhage of any extent in the tumor resection cavity was evident in close to a third of cases; only 3 of these required surgical evacuation secondary to concerns regarding mass effect. The percentage of postoperative seizures was lower than preoperative sei-

TABLE 2. A comparison of perioperative patient and tumor characteristics among skull base and non-skull base meningiomas

\begin{tabular}{lcc}
\hline \multicolumn{1}{c}{ Variable } & Non-Skull Base & Skull Base \\
\hline No. of patients & 269 & 129 \\
\hline Mean age \pm SD (yrs) & $58.3 \pm 15.3$ & $55.15 \pm 13.3$ \\
\hline Males (\%) & $95(35)$ & $41(32)$ \\
\hline Mean follow-up (mos) & 40 & 51.5 \\
\hline WHO Grade (\%) & & \\
I & $186(69.1)$ & $110(85.3)$ \\
II & $63(23.4)$ & $15(11.6)$ \\
III & $19(7.1)$ & $4(3.1)$ \\
$\quad$ Unknown & $1(0.4)$ & 0 \\
\hline Primary presentation at initial op (\%) & $243(90.3)$ & $116(89.9)$ \\
\hline Average MIB-1 index for initial & $0.062 \pm 0.061$ & $0.04 \pm 0.027$ \\
$\quad$ tumor \pm SD & $84(31.2)$ & $41(31.8)$ \\
\hline STR (\%) & $46(17.1)$ & $9(7.0)$ \\
\hline Postop radiation (\%) & $46(17.1)$ & $17(13.2)$ \\
\hline Recurrence (\%) & 47.3 & 31.8 \\
\hline Median time to recurrence (mos) &
\end{tabular}


zures (17.6\% vs $23.9 \%$, no significant differences); perioperative seizure prophylaxis was used in approximately $10 \%$ of patients.

\section{Skull Base and Non-Skull Base Meningiomas}

A patient's sex and the ability to obtain a GTR were independent of tumor location. Patients with skull base meningiomas were younger at the time of surgery $(55.2$ vs 58.3 years, respectively; $p=0.01$ ), had a significantly higher proportion of WHO Grade I meningiomas (85.3\% vs $69.1 \%$, respectively; $p=0.003$ ), and had a lower mean MIB-1 index (0.041 vs 0.062, respectively; $p=0.001$ ). Patients with non-skull base meningiomas were more likely to undergo postoperative radiation therapy $(17.1 \%$ vs $7 \%$, $\mathrm{p}=0.006$ ), likely a reflection of the higher WHO grade of tumors in this cohort. While the median time to recurrence was shorter in the cohort of skull base meningiomas (31.8 months vs 47.3 months), this was not statistically significant. Among non-skull base tumors, the occurrence of WHO Grade II/III tumors was higher among meningiomas located in the parasagittal and falcine regions (11 of $24,46 \%$ ) compared with the other locations (63 of 244 , $26 \%$; Table 3 ); the odds ratio (OR) for this correlation was 2.2 and approached significance $(95 \%$ confidence interval $[\mathrm{CI}]$ 0.94-5.2, $\mathrm{p}=0.07$ ). A comparison of the WHO grade of sphenoid ridge meningiomas suggested that $21 \%$ of lateral and 50\% of medial tumors were of higher WHO grades. However, due to the very small sample of patients with distinctly medial tumors (4 total), this relationship was not statistically significant (OR 3.8, 95\% CI 0.4-34.1; $\mathrm{p}=0.23$ ).

\section{Odds of Overall Recurrence}

\section{Prior History of Recurrence}

Patients who had previously undergone surgical intervention, prior to first presenting at our institution, had significantly higher odds of tumor recurrence compared with patients undergoing surgical intervention for the first time (OR 6.24, 95\% CI 3.0-12.7, p < 0.001; Fig. 1).

\section{Extent of Resection}

Based on a pooled sample of patients representing meningiomas in all locations, the 10 -year recurrence-free survival rate in patients with GTR was $90 \%$, while in patients for whom an STR was obtained it was 43\%. This difference in odds was statistically significant (OR 6.74, 95\% CI 3.6-12.3, p < 0.001; Fig. 2).

\section{Meningioma Location}

Patients with skull base and non-skull base meningiomas were found to have a similar rate of recurrence until approximately 100 months of overall follow-up (Fig. 3 ); subsequent to this period, however, while the recurrence of non-skull base meningiomas persisted at the same rate, the pattern of skull base meningiomas demonstrated a plateau (Fig. 3A). However, this was based on 65 total patients ( 45 with non-skull base and 20 with skull base meningiomas). An analysis of the skull base versus non-skull base cohorts following the 100-month follow-up period demonstrated that the difference in the recurrence pattern was statistically significant. A sepa-

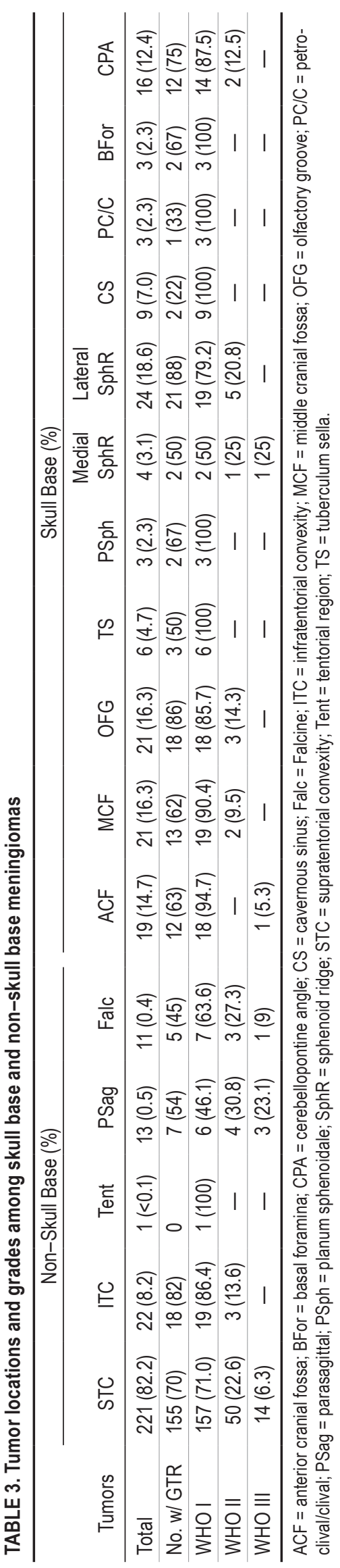




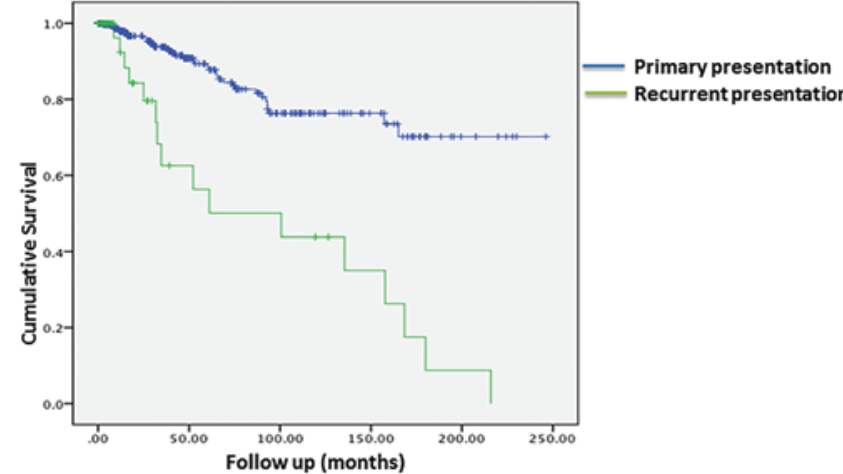

FIG. 1. Kaplan-Meier curve showing overall pattern of recurrence based on a prior history of recurrence. Figure is available in color online only.

rate analysis of only patients with a primary presentation to our institution (243 non-skull base and 116 skull base patients) showed no substantial difference regarding this trend (data not shown). Focusing solely on WHO Grade I tumors (186 with non-skull base and 110 with skull base tumors) showed a similar diverging recurrence trend, although this occurred at approximately 150 months (data not shown). To account for the possibility of the EOR affecting recurrence patterns, Kaplan-Meier analysis was conducted separately in the subcohorts of skull base/ non-skull base meningiomas with a GTR (Fig. 3B) and STR (Fig. 3C). Although patients with an STR, regardless of location, had a higher recurrence rate, the pattern of divergence within the 50-100-month follow-up period between skull base and non-skull base meningiomas remained consistent. To account for the possibility of the higher proportion of WHO Grade I tumors among skull base meningiomas affecting recurrence patterns, KaplanMeier curves were constructed for Grade I meningiomas in both locations, stratified by GTR and STR (Fig. 3D and E, respectively); Figure 3F demonstrates the Kaplan-Meier curves for recurrence patterns based on location, stratified by GTR and WHO Grades II/III. The overall diverging patterns of recurrence were maintained.

\section{WHO Grade}

Overall, WHO Grade III tumors had a significantly higher likelihood of recurrence following resection $(\mathrm{p}<$ 0.001, Fig. 4A). This was also dependent on the EOR: regardless of grade, the recurrence rate of all tumors was lower in cases of GTR compared with STR. In GTR cases, WHO Grade I tumors were significantly less likely to recur compared with Grade II and III tumors ( $\mathrm{p}=0.03$, Fig. 4B), while in cases with STR, Grade I and II tumors followed a similar trend, which was different from the pattern noted with Grade III tumors ( $p=0.002$, Fig. 4C). Caution must be advised in interpreting these results, however, given the smaller sample size of patients with Grade II and III tumors compared with Grade I tumors.

\section{Statistical Modeling to Predict Overall Patterns of Recurrence}

Regression analysis was conducted to identify potential predictors of overall recurrence. On univariate analysis,

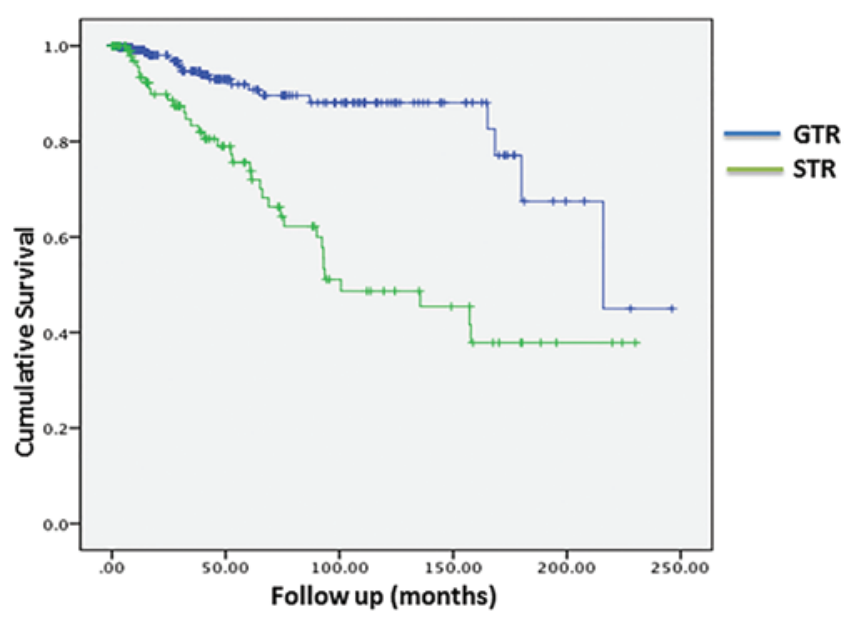

FIG. 2. Kaplan-Meier curve of the overall pattern of recurrence based on EOR. Figure is available in color online only.

the following factors were found to influence the recurrence of meningiomas: a prior history of recurrence $(p$ $<0.001$ ), initial WHO grade following resection at our center ( $\mathrm{p}<0.001$ ), and the inability to obtain GTR ( $\mathrm{p}<$ 0.001). While tumor location was overall not significant $(p=0.25)$, in the cohorts of patients followed longer than 100 months, patients with a tumor in the skull base were significantly less likely to experience a recurrence $(\mathrm{p}=$ 0.012; upon applying Bonferroni correction for multiple comparisons, $\mathrm{p}=0.025$ ). Age, sex, perioperative seizure status, tumor diameter, volume of tumor, remote history of radiation, PR status, or any history of hormone replacement therapy/oral contraceptive medication use were not significant predictors of recurrence. The presence of hemorrhage in the tumor resection cavity $(\mathrm{p}=0.002)$ and the volume of peritumoral edema $(\mathrm{p}=0.037)$ were significant predictors while the MIB-1 index $(\mathrm{p}=0.1)$ approached significance. Postoperative hemorrhage and edema were not correlated with tumor grade. Due to colinearity and a small sample size, these 3 latter variables could not be incorporated into the multivariate model.

In the multivariate Cox regression model, a prior history of recurrence $(p=0.02)$ and the inability to obtain a GTR $(\mathrm{p}<0.01)$ remained as significant factors associated with recurrence. The predictors of recurrence were further assessed through an analysis that focused solely on patients with a primary presentation. In this analysis, the inability to obtain GTR and tumors with WHO Grades II and III were both predictors of a more rapid recurrence ( $p$ $<0.001$ for both variables).

\section{Discussion}

In this study we have analyzed one of the largest cohorts of patients with intracranial meningiomas who have undergone resection and were followed postoperatively for a relatively long period. The patients included in the time period of analysis closely resemble the cohort of patients that are managed and followed in modern-day neurosurgical practice. In this population, WHO grade, prior evidence of recurrence, and an inability to achieve GTR were 

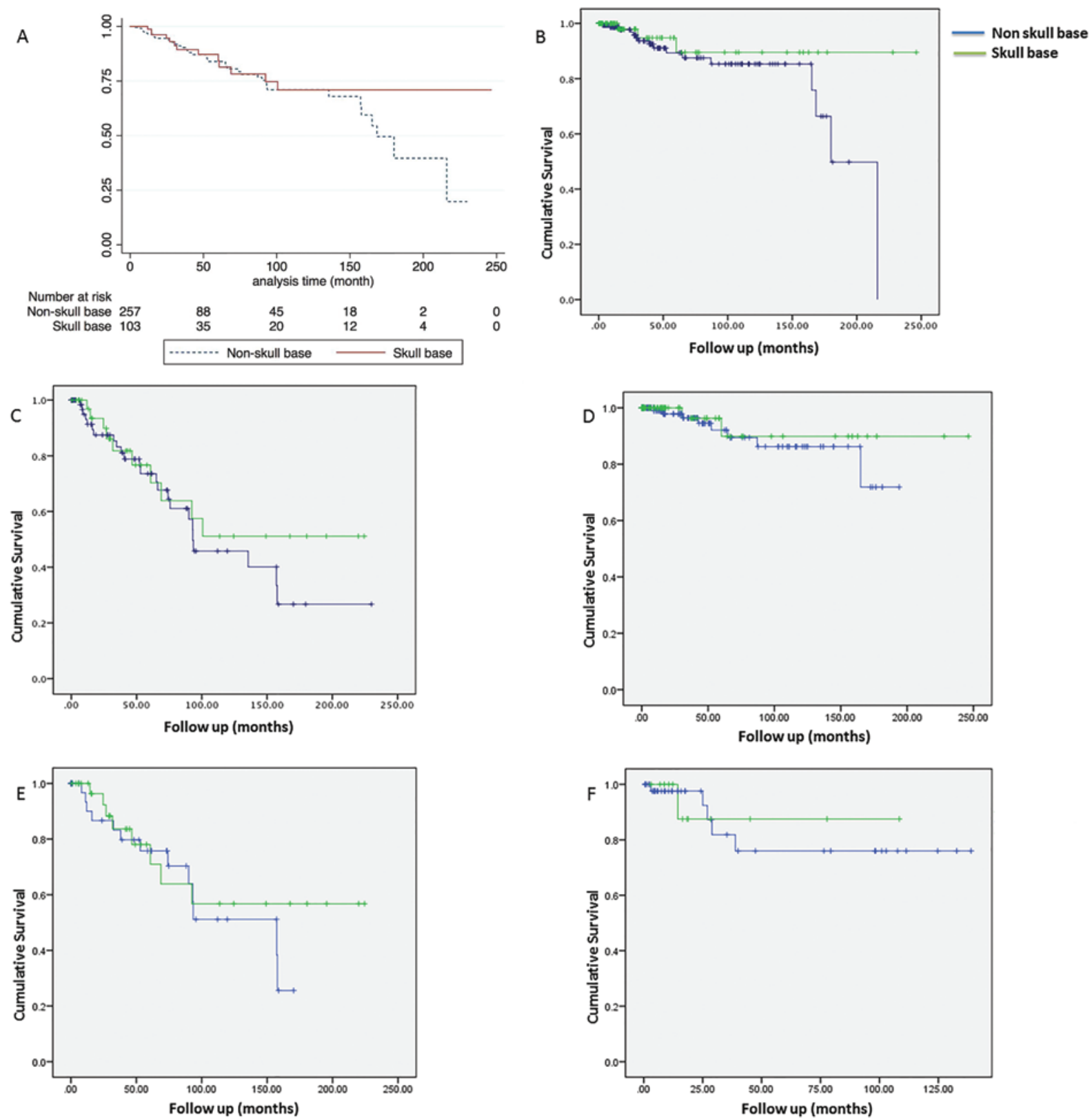

FIG. 3. Kaplan-Meier curves of the pattern of recurrence based on location: overall sample (A); stratified by GTR (B); stratified by STR (C); based on location, stratified by GTR and WHO Grade I (D); based on location, stratified by STR and WHO Grade I (E); and based on location, stratified by GTR and WHO Grade II and III (F). Figure is available in color online only.

significant predictors of overall postoperative recurrence; the latter two factors were found to be independent predictors of recurrence based on multivariate analysis. Given that patients with a prior history of tumor recurrence likely had higher-grade tumors, the elimination of this cohort of patients resulted in both the EOR and the WHO grade becoming significant predictors of recurrence. Through a comparison of patients with skull base and non-skull base intracranial meningiomas as separate cohorts, we have demonstrated that patients with skull base meningiomas require surgical intervention at a significantly younger age, but their tumors have a significantly lower average MIB-1 index and are more likely to be WHO Grade I. This corre- lation is in agreement with various recent studies suggesting the benign nature of skull base meningiomas in comparison with superficial ones. ${ }^{7,13,15}$ Furthermore, long-term follow-up of our patients suggests that non-skull base meningiomas demonstrate a continued pattern of recurrence, whereas skull base meningiomas plateau beyond 100 months; this divergence beyond 100 months was shown to be statistically significant, although based on a smaller cohort of patients. These results have potential implications for postoperative decision-making, long-term follow-up of patients subsequent to surgical intervention, and predicting the possibility of the need for a second intervention.

Most of the data regarding the long-term postoperative 

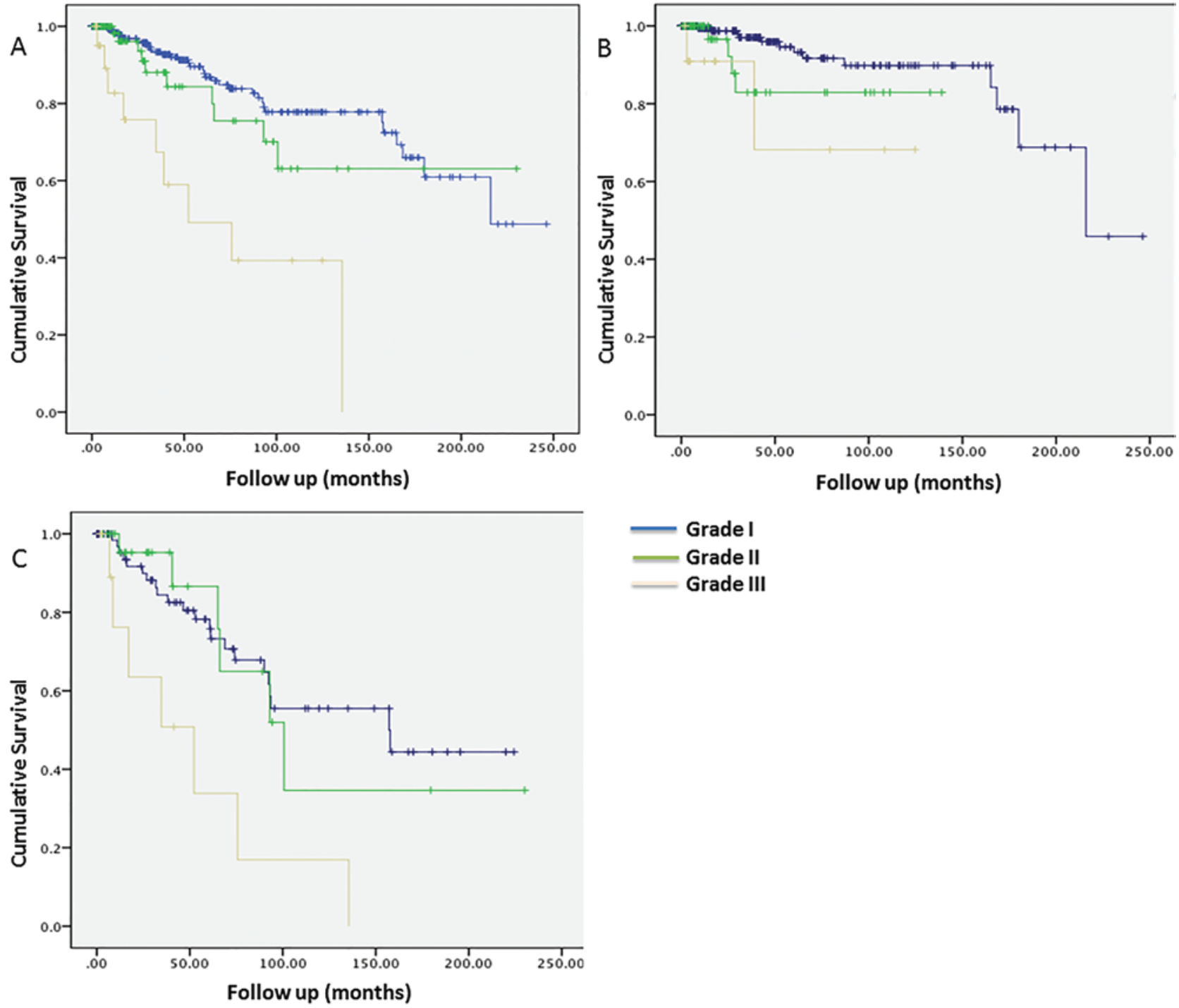

FIG. 4. Kaplan-Meier curves showing the pattern of recurrence based on WHO Grade, in the overall cohort (A), stratified by GTR (B), and stratified by STR (C). Figure is available in color online only.

recurrence rates of meningiomas in various intracranial locations have been based on surgical series prior to the era of neuronavigation and other surgical adjuncts. In one of the earliest series published in 1983, Adegbite et al. surgically managed 114 intracranial meningiomas. ${ }^{1}$ Evidence of tumor recurrence, the criteria for which were not defined, was noted in $19 \%$ of cases and the overall recurrence-free survival rate was noted to be $80 \%$ at 5 years. The authors found the Simpson grade of initial resection to be the only significant predictor of recurrence; a multivariate analysis was not conducted to assess the possible interaction of other variables. In a larger series of 276 patients with a mean follow-up of 5.1 years published in 1994, Mahmood et al. showed a mean time to recurrence of 46 months and an approximately 93\% recurrence-free survival rate at 5 years. ${ }^{17}$ The authors showed a significantly lower rate of recurrence in cases of GTR compared with STR, regardless of location. The reported recurrence rate in this study was $6 \%$ overall, which is likely due to a higher proportion of benign tumors ( 254 of 276 samples), the frequency of imaging follow-up, and the sensitivity of the available imaging modality at the time. The authors suggested that the majority of tumors that are typically classified as recurrences in essence represent evidence of tumor "re-growth" and that only higher-grade tumors demonstrate true recurrences. The overall conclusions of this study were similar to the observation in our sample of patients, wherein EOR was an independent predictor of recurrence, but contrary to results in other studies that suggest that a skull base location is a predictor of higher recurrence, ${ }^{19,24,30}$ this is likely an attribute of the difficulty in obtaining GTR for skull base tumors in these older series, perhaps due to sensitivity of imaging analysis and availability of surgical adjuncts such as neuronavigation.

In 1996, Mathiesen and colleagues assessed a surgical cohort of patients with skull base meningiomas; the mean follow-up was 18 years (spanning the years 1947 to 1982). ${ }^{19}$ Similar to our study, the majority of these lesions did not 
demonstrate malignant features and the majority of recurrences were within the first 10 years following surgery. This study was the first to convey the need for long-term postoperative follow-up of patients with meningioma. However, the $10.8 \%$ perioperative mortality rate, likely an attribute of limited access to high-resolution imaging and surgical adjuncts (e.g., neuronavigation, ultrasonic aspirators, and higher quality bipolar cautery, to name a few examples), ${ }^{31}$ was concerning. Furthermore, this study also had not included relevant intrinsic biological properties of tumors, such as the proliferation index.1,4,12,18,21 In our current study, representing a relatively more modern series of patients, we have included and confirmed the relevance of the MIB-1 index and the WHO grade as important variables affecting recurrence, as had been commonly recognized in prior recent studies.

The MIB-1 index of proliferation has been used extensively for the assessment of proliferative activity in meningiomas. ${ }^{16,27,28}$ In an analysis on WHO Grade II and III meningiomas, a higher overall MIB-1 index was identified in recurrent tumors. ${ }^{2}$ In a study of 344 meningiomas (157 in the skull base), a non-skull base location was shown to be an independent predictor of a higher MIB-1 index. ${ }^{14}$ In an analysis of 2 separate cohorts, one with incidentally diagnosed meningiomas and the other with resected meningiomas, Hashimoto and colleagues demonstrated that skull base meningiomas had a lower rate of growth in the former cohort (38 skull base and 75 non-skull base meningiomas) and a lower average MIB-1 index in the latter cohort (94 skull base and 116 non-skull base meningiomas). ${ }^{9}$ Given the parallel nature of this analysis, it is difficult to draw definitive conclusions with regard to the correlation of MIB-1 index and patterns of growth postoperatively. Furthermore, only the MIB-1 index of WHO Grade I meningiomas was assessed. McGovern and colleagues reported their institutional data on 216 patients with meningiomas who were surgically managed and followed for a median of 7.2 years. In an assessment of 183 resected WHO Grade I meningiomas (71 skull base, 93 non-skull base), the authors observed that non-skull base meningiomas had a significantly higher MIB-1 index but a lower rate of recurrence. ${ }^{20}$ In this study, the rate of GTR in skull base meningiomas was $41 \%$, while the rate for non-skull base meningiomas was $78 \%$. In our study, the EOR was similar regardless of location (STR in $31.2 \%$ of non-skull base and $31.8 \%$ in skull base). Furthermore, the EOR and the WHO grade of the tumor were shown to be influential in tumor recurrence in our cohort of patients. Therefore, discrepancies between our findings and those of McGovern et al. could potentially stem from differential EOR and the restriction of the latter analysis to WHO Grade I meningiomas.

Although both the WHO grade and the MIB-1 index were significantly different in skull base and non-skull base lesions, only the WHO grade (when eliminating patients with a prior history of recurrence) was an independent predictor of recurrence in our series. The correlation of MIB-1 proliferation index with tumor grade, and the relatively small sample size of patients, are likely explanations. Alternatively, the histopathological variables MIB-1 index and PR status are most likely not reflective of tumor biology and/or clinical behavior. The defining biological features of meningiomas are certainly more complex and future identification of such biological markers will be necessary.

While the grading of the MIB-1 index assists in communicating the likely aggressiveness of a tumor, this tool may be subjected to limits in its reproducibility due to a lack of standardized methods of immunostaining, heterogeneity of tumor regions, and other issues with assessment of staining pattern. ${ }^{23,28}$ This has led to variability of procedures and reference values used across different institutions. ${ }^{28}$ Therefore, the Clinical Neuropathology practice guide was unable to recommend its routine implementation for prognostication purposes ${ }^{3}$ and it is difficult to envision that the MIB-1 index alone would be a suitable method for predicting recurrence; other strategies are necessary. One such recently established strategy has been the detection of phosphohistone H3 as a more robust method of calculating mitotic index and correlating it with recurrence-free survival. ${ }^{26}$ Other studies have demonstrated the potential correlation of tumor suppressor genes (e.g., DAL-1 [progression], TIMP-1 [tumor invasion], and $N D R G 2$ [tumor recurrence] $)^{8,22,25}$ and aberrations in signaling pathways (e.g., Wnt [tumor invasion and recurrence] $)^{34}$ with aggressive tumor behavior in meningiomas. Furthermore, genome-sequencing studies have identified additional novel mutations associated with certain meningioma types. ${ }^{5,7}$ While further confirmatory studies are necessary, it is highly likely that future studies assessing the long-term recurrence patterns of meningiomas would need to consider a more exhaustive list of variables that define the biological features of meningiomas.

The limitations of this study are those that are wellknown for retrospective investigations and these include difficulties with ascertaining the fate of missing data, inability to obtain detailed Simpson grading for tumors resected, nonstandardized reporting of MIB-1 indices, and an inability to access imaging for all patients at every follow-up. Furthermore, given the time span over which our study was conducted, the imaging modalities, operative techniques, and postoperative adjunctive therapies were not uniform. While this may have introduced heterogeneity, this choice was relevant given that many of the patients in the current neurosurgical practice may have been managed surgically several years prior. Furthermore, and related to limitations in accessing all imaging media, volumetric measurements could not be made for all patients. Also, given the tertiary referral nature of our center, it is likely that many of the lesions managed would be typically classified as more challenging from a surgical point of view. This limits the generalizability of our findings to perhaps a specific cohort of patients. In addition, it must be emphasized that the observation regarding the diverging recurrence pattern is driven by a smaller sample of the overall cohort. Nonetheless, this study represents one of the largest modern series of patients with surgically managed meningiomas followed for a relatively long duration. In light of emerging evidence with regard to the more benign nature of skull base meningiomas, the overall concept of divergent recurrence patterns is likely true but must be systematically explored in future surgical series as well. 
In this study, the importance of the EOR and the WHO grade as predictors of recurrence has been reemphasized. Furthermore, our findings have added strength to the theory that skull base meningiomas are biologically different and perhaps less aggressive than non-skull base meningiomas. Our observation with regard to the 100-month period of diverging patterns of recurrence between meningiomas in the two locations and its persistence despite accounting for difference in the EOR is noteworthy. It is possible that the influence of EOR pertains to the period before this divergence, while the inherent biology of the tumor is responsible for recurrence (or perhaps regrowth, as suggested by Mahmood et al. ${ }^{17}$ ) beyond this 100 -month period. The traditional features of tumor biology could not account for this dramatic difference, as the diverging pattern was maintained when stratifying by GTR and high WHO grade, suggesting that alternative molecular, genetic, and perhaps epigenetic features of meningiomas should be accounted for in future studies assessing the long-term postoperative pattern of tumor recurrence.

\section{Acknowledgments}

We would like to acknowledge the contribution of Mr. Shahin Khayyer in the preparation of the figures.

\section{References}

1. Adegbite AB, Khan MI, Paine KW, Tan LK: The recurrence of intracranial meningiomas after surgical treatment. J Neurosurg 58:51-56, 1983

2. Al-Mefty O, Kadri PA, Pravdenkova S, Sawyer JR, Stangeby $\mathrm{C}$, Husain M: Malignant progression in meningioma: documentation of a series and analysis of cytogenetic findings. $\mathbf{J}$ Neurosurg 101:210-218, 2004

3. Berghoff AS, Stefanits H, Woehrer A, Heinzl H, Preusser M, Hainfellner JA: Clinical neuropathology practice guide 3-2013: levels of evidence and clinical utility of prognostic and predictive candidate brain tumor biomarkers. Clin Neuropathol 32:148-158, 2013

4. Borovich B, Doron Y: Recurrence of intracranial meningiomas: the role played by regional multicentricity. J Neurosurg 64:58-63, 1986

5. Brastianos PK, Horowitz PM, Santagata S, Jones RT, McKenna A, Getz G, et al: Genomic sequencing of meningiomas identifies oncogenic SMO and AKT1 mutations. Nat Genet 45:285-289, 2013

6. Christensen D, Laursen H, Klinken L: Prediction of recurrence in meningiomas after surgical treatment. A quantitative approach. Acta Neuropathol 61:130-134, 1983

7. Clark VE, Erson-Omay EZ, Serin A, Yin J, Cotney J, Ozduman K, et al: Genomic analysis of non-NF2 meningiomas reveals mutations in TRAF7, KLF4, AKT1, and SMO. Science 339: 1077-1080, 2013

8. Halaka AN, Bunning RA, Bird CC, Gibson M, Reynolds JJ: Production of collagenase and inhibitor (TIMP) by intracranial tumors and dura in vitro. J Neurosurg 59:461-466, 1983

9. Hashimoto N, Rabo CS, Okita Y, Kinoshita M, Kagawa N, Fujimoto Y, et al: Slower growth of skull base meningiomas compared with non-skull base meningiomas based on volumetric and biological studies. J Neurosurg 116:574-580, 2012

10. Hayhurst C, Zadeh G: Tumor pseudoprogression following radiosurgery for vestibular schwannoma. Neuro Oncol 14:87-92, 2012

11. Hsu DW, Efird JT, Hedley-Whyte ET: Progesterone and es- trogen receptors in meningiomas: prognostic considerations. J Neurosurg 86:113-120, 1997

12. Jääskeläinen J: Seemingly complete removal of histologically benign intracranial meningioma: late recurrence rate and factors predicting recurrence in 657 patients. A multivariate analysis. Surg Neurol 26:461-469, 1986

13. Kane AJ, Sughrue ME, Rutkowski MJ, Shangari G, Fang $\mathrm{S}, \mathrm{McDermott} \mathrm{MW}$, et al: Anatomic location is a risk factor for atypical and malignant meningiomas. Cancer 117:12721278,2011

14. Kasuya H, Kubo O, Tanaka M, Amano K, Kato K, Hori T: Clinical and radiological features related to the growth potential of meningioma. Neurosurg Rev 29:293-297, 2006

15. Ketter R, Rahnenführer J, Henn W, Kim YJ, Feiden W, Steudel WI, et al: Correspondence of tumor localization with tumor recurrence and cytogenetic progression in meningiomas. Neurosurgery 62:61-70, 2008

16. Langford LA, Cooksley CS, DeMonte F: Comparison of MIB-1 (Ki-67) antigen and bromodeoxyuridine proliferation indices in meningiomas. Hum Pathol 27:350-354, 1996

17. Mahmood A, Qureshi NH, Malik GM: Intracranial meningiomas: analysis of recurrence after surgical treatment. Acta Neurochir (Wien) 126:53-58, 1994

18. Marks SM, Whitwell HL, Lye RH: Recurrence of meningiomas after operation. Surg Neurol 25:436-440, 1986

19. Mathiesen T, Lindquist C, Kihlström L, Karlsson B: Recurrence of cranial base meningiomas. Neurosurgery 39:2-9, 1996

20. McGovern SL, Aldape KD, Munsell MF, Mahajan A, DeMonte F, Woo SY: A comparison of World Health Organization tumor grades at recurrence in patients with non-skull base and skull base meningiomas. J Neurosurg 112:925933, 2010

21. Mirimanoff RO, Dosoretz DE, Linggood RM, Ojemann RG, Martuza RL: Meningioma: analysis of recurrence and progression following neurosurgical resection. J Neurosurg 62:18-24, 1985

22. Mizoue T, Kawamoto H, Arita K, Tominaga A, Kurisu K: Secretion of matrix metalloproteinase- 9 and tissue inhibitor of metalloproteinase-1 by meningiomas detected by cell immunoblot analysis. Acta Neurochir (Wien) 141:481-486, 1999

23. Morimura T, Kitz K, Budka H: In situ analysis of cell kinetics in human brain tumors. A comparative immunocytochemical study of S phase cells by a new in vitro bromodeoxyuridine-labeling technique, and of proliferating pool cells by monoclonal antibody Ki-67. Acta Neuropathol 77:276-282, 1989

24. Nanda A, Vannemreddy P: Recurrence and outcome in skull base meningiomas: do they differ from other intracranial meningiomas? Skull Base 18:243-252, 2008

25. Nunes F, Shen Y, Niida Y, Beauchamp R, Stemmer-Rachamimov AO, Ramesh V, et al: Inactivation patterns of NF2 and DAL-1/4.1B (EPB41L3) in sporadic meningioma. Cancer Genet Cytogenet 162:135-139, 2005

26. Olar A, Wani KM, Sulman EP, Mansouri A, Zadeh G, Wilson $\mathrm{CD}$, et al: Mitotic index is an independent predictor of recurrence-free survival in meningioma. Brain Pathol 25:266-275, 2015

27. Prayson RA: Malignant meningioma: a clinicopathologic study of 23 patients including MIB1 and p53 immunohistochemistry. Am J Clin Pathol 105:719-726, 1996

28. Prayson RA: The utility of MIB-1/Ki-67 immunostaining in the evaluation of central nervous system neoplasms. Adv Anat Pathol 12:144-148, 2005

29. Sade B, Chahlavi A, Krishnaney A, Nagel S, Choi E, Lee JH: World Health Organization Grades II and III meningiomas are rare in the cranial base and spine. Neurosurgery 61:1194-1198, 2007 
30. Simpson D: The recurrence of intracranial meningiomas after surgical treatment. J Neurol Neurosurg Psychiatry 20:22-39, 1957

31. Sughrue ME, Kane AJ, Shangari G, Rutkowski MJ, McDermott MW, Berger MS, et al: The relevance of Simpson Grade I and II resection in modern neurosurgical treatment of World Health Organization Grade I meningiomas. J Neurosurg 113:1029-1035, 2010

32. Wiemels J, Wrensch M, Claus EB: Epidemiology and etiology of meningioma. J Neurooncol 99:307-314, 2010

33. Yushkevich PA, Piven J, Hazlett HC, Smith RG, Ho S, Gee JC, et al: User-guided 3D active contour segmentation of anatomical structures: significantly improved efficiency and reliability. Neuroimage 31:1116-1128, 2006

34. Zhou K, Wang G, Wang Y, Jin H, Yang S, Liu C: The potential involvement of E-cadherin and beta-catenins in meningioma. PLoS One 5:e11231, 2010

\section{Disclosures}

The authors report no conflict of interest concerning the materi- als or methods used in this study or the findings specified in this paper.

\section{Author Contributions}

Conception and design: Mansouri, Aldape, Zadeh. Acquisition of data: Mansouri, Klironomos, Taslimi, Kilian, Khan. Analysis and interpretation of data: Mansouri, Taslimi, Aldape. Drafting the article: Mansouri, Klironomos, Zadeh. Critically revising the article: Mansouri, Klironomos, Aldape, Zadeh. Reviewed submitted version of manuscript: all authors. Approved the final version of the manuscript on behalf of all authors: Mansouri. Statistical analysis: Mansouri, Taslimi. Administrative/technical/material support: Aldape, Zadeh. Study supervision: Gentili, Aldape, Zadeh.

\section{Correspondence}

Alireza Mansouri, Toronto Western Hospital, 4W-436, 399 Bathurst St., Toronto, ON M5T 2S8, Canada. email: alireza. mansouri@utoronto.ca. 